\title{
Anti-GABAB receptor antibodies with autoimmune encephalitis in clear cell renal cell carcinoma: a case report
}

\author{
Huanrui Wang, Weiyu Zhang, Xiaopeng Zhang, Tao Xu, Kexin $\mathrm{Xu}^{\wedge}$ \\ Department of Urology, Peking University People's Hospital, Beijing, China \\ Correspondence to: Kexin Xu. Department of Urology, Peking University People's Hospital, No. 11 XiZhiMen South Street, Beijing, 100044, China. \\ Email: cavinx@yeah.net.
}

\begin{abstract}
Encephalitis with anti- $\gamma$-aminobutyric acid-B (GABAB) receptor antibodies is an autoimmune encephalitis, which is mainly observed with small-cell lung cancer (SCLC), thymoma, and melanoma. Here, we reported a case of clear cell renal cell carcinoma with the loss of consciousness as a first symptom, which was associated with a rare GABAB receptor antibody limbic encephalitis. A 58-year-old man with a 2-day history of stomachache and unconsciousness. Imaging studies of the head were normal. The abdominal computed tomography revealed a $3.9 \mathrm{~cm} \times 3.8 \mathrm{~cm}$ right renal mass with contrast enhanced. Subsequent cerebrospinal fluid antibody testing was positive for anti-GABAB receptor antibodies. After 2 weeks of treatment with nutritional neurologic and immunomodulatory, the symptoms did not improve. Laparoscopic radical nephrectomy was undertaken and subsequently immunotherapy for the patient's treatment. The postoperative pathology was renal cell carcinoma (Clear cell carcinoma, WHO/ISUP grade 3, tumor size $4 \mathrm{~cm} \times 3.5 \mathrm{~cm}, \mathrm{pT} 1 \mathrm{a})$. The patient's conditions improved after the surgery. At the 12-month follow-up, computed tomography imaging showed no recurrence, and the patient was living independently. This case was reported to demonstrate that, when patients are presented with GABAB receptor antibody encephalitis, early evaluation of underlying malignancy including renal cell carcinoma and aggressive treatment of primary tumors provide the chances for a better outcome.
\end{abstract}

Keywords: Autoimmune encephalitis; $\gamma$-aminobutyric acid-B (GABAB) receptor; renal cell carcinoma; laparoscopic radical nephrectomy; case report

Submitted Aug 21, 2020. Accepted for publication Dec 28, 2020.

doi: $10.21037 /$ tcr-20-2769

View this article at: http://dx.doi.org/10.21037/tcr-20-2769

\section{Introduction}

Anti- $\gamma$-aminobutyric acid-B (GABAB) receptor encephalitis was first described by Lancaster E in 2010 (1). Approximately $50 \%$ of patients with anti-GABAB encephalitis had tumors, most frequently arise in small-cell lung cancer (SCLC) $(1,2)$. The following tumors has been reported in a few cases associated with anti-GABAB receptor encephalitis: adenocarcinoma, melanoma, esophageal cancer, myeloma. It is no clear whether other tumors are related to this condition. Herein, we present a rare case of anti-GABAB receptor encephalitis associated with renal cell carcinoma, where the patient showed loss of consciousness and epilepsy as first symptoms.

We present the following article in accordance with the CARE reporting checklist (available at http://dx.doi. org/10.21037/tcr-20-2769).

\section{Case presentation}

A male patient aged 58 was admitted to the emergency department with a 2-day history of stomachache and

^ ORCID: 0000-0003-1057-1467. 

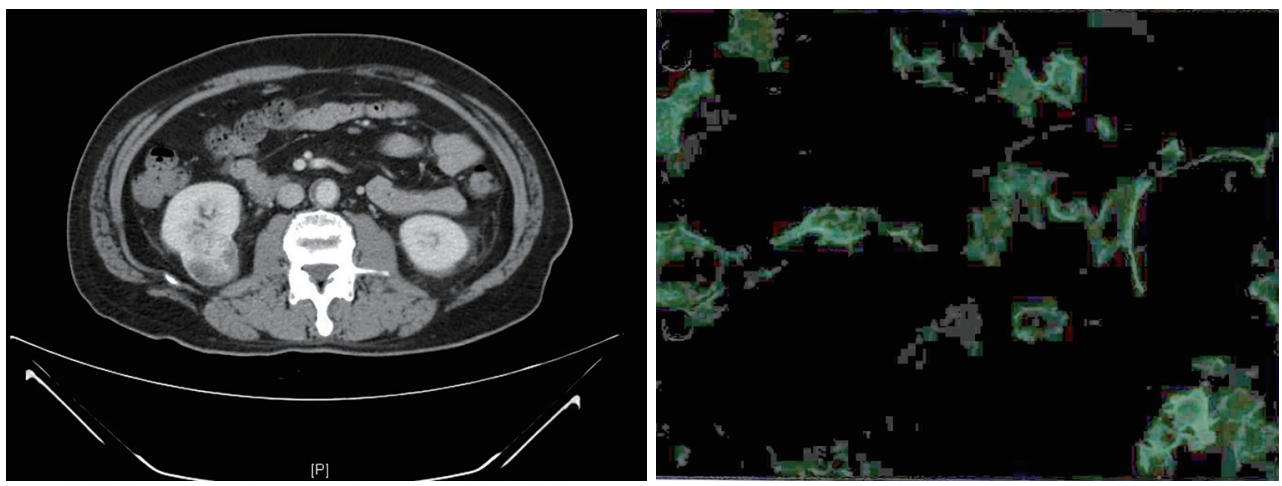

Figure 1 Computed tomography image and cerebrospinal fluid analysis. (A) A computed tomography image showing renal cell carcinoma in the right kidney. (B) Cerebrospinal fluid anti-GABABR antibodies transfected cells image (immunofluorescence staining; $\times 400$ ).

unconsciousness on Nov. 07, 2018. There were no obvious abnormalities in the brain $\mathrm{CT}$ without contrast. The abdominal CT revealed a $3.9 \mathrm{~cm} \times 3.8 \mathrm{~cm}$ right renal mass with contrast enhanced (Figure 1A). The electrocardiogram showed sinus tachycardia with a heart rate of $123 \mathrm{bpm}$. Routine blood test: WBC: $25.34 \times 10^{9} / \mathrm{L}, \mathrm{NE} \%$ : $83.6 \%$. Ornidazole $40 \mathrm{mg}$ anti-infection, phloroglucinol $60 \mathrm{mg}$ relief gastrointestinal spasm. The patient experienced generalized seizure again with decreasing oxygen saturation level to $83 \%$ during the treatment. He was treated with emergency tracheal intubation immediately. The patient was then referred to Peking University Peoples' Hospital for further assessment and treatment. The evaluation of CSF sample presented normal intracranial pressure $\left(110 \mathrm{mmH}_{2} \mathrm{O}\right), 7$ leukocytes, total protein $520 \mathrm{mg} / \mathrm{L}$, glucose $4.88 \mathrm{mmol} / \mathrm{L}$, normal chlorine level $(125.7 \mathrm{mmol} / \mathrm{L})$. The analyse results of the viral serological and CSF were negative (rubella virus, cytomegalovirus, herpes simplex viruses, and Epstein-Barr virus). Serum tumor markers including CEA, AFP, CA19-9, CYFRA21-1, NSE, ProGR, and tPSA in serum were all negative. Abnormal wave, single or rhythmic moderate slow wave sharp wave rhythm or complex wave on the right frontal pole and the frontal mid-temporal zone, the mid-range slow wave can be seen in the full conductance sometimes, and the left hemisphere has a higher amplitude, especially in the left frontal area, was found in electroencephalogram (EEG). After 2 weeks of treatment with nutritional neurologic and immunomodulatory, the symptoms did not improve. There was possibility of Paraneoplastic limbic encephalitis (PLE) associated with malignant tumor in the right kidney. The paraneoplastic antibodies in CSF were also under analysis. Only GABAB antibodies were positive in the CSF
(Figure 1B).

Considering the patient has paraneoplastic autoimmune encephalitis of kidney cancer, conservative treatment is less likely to help regain consciousness, and the primary tumor needed to be resected as soon as possible. Laparoscopic radical nephrectomy surgery was successfully performed. The postoperative pathology was Renal Cell Carcinoma (Clear cell carcinoma, WHO/ISUP grade 3, tumor size $4 \mathrm{~cm} \times 3.5 \mathrm{~cm}, \mathrm{pT} 1 \mathrm{a}, \mathrm{pNx}$ ) (Figure 2). Gammaglobulin combined with metacortandracin was administered after the surgery. Seizures stopped after the initiation of levetiracetam. The patient reported no irritability, no seizures, but partial memory loss 2 months after the surgery. At the 12-month follow-up, the patient had no recurrence and experienced significant improvement in memory. The patient had only mild cognitive impairment and was living independently (MRS, 2/6).

All procedures performed in studies involving human participants were in accordance with the ethical standards of the institutional and/or national research committee(s) and with the Helsinki Declaration (as revised in 2013). Written informed consent was obtained from the patient.

\section{Discussion}

Autoimmune encephalitides is a syndrome characterized by subacute amnesia, epilepsy, and mental symptoms (3). Lancaster E reported autoimmune encephalitis associated with GABAbR-Abs in a case series of 20 patients in 2010 (1). GABA-B receptor involved in the glutamate regulation system acting as a pre- and post-synaptic receptor (4). Seizures accrues early and prominently in GABAb patients, and $50 \%$ of this patient population have an underlying 

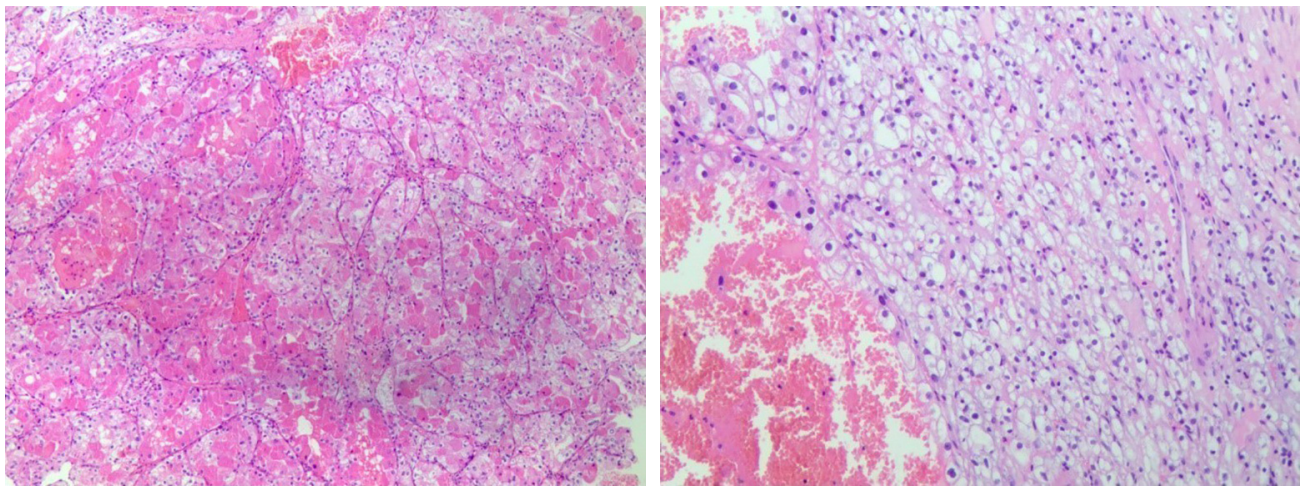

Figure 2 Hematoxylin and eosin-stained sections showing clear cell renal cell carcinoma (A, 100×, B, 200×).

malignant carcinoma, most frequently small cell lung cancer (2). We reported a clinical course of a middleaged patient with kidney cancer and anti-GABAB receptor encephalitis. The initial symptoms were recurring seizures, subsequently loss of memory and consciousness, consistent with limbic encephalitis. It remains unknown whether there is a causality between RCC and the presence of GABAB antibodies. Coincidentally, it has been reported that, GABA was associated with prognosis among patients with ccRCC (5). Per the report, GABAbR-Abs encephalitis is unassociated with malignant carcinoma in about $40 \%$ of cases $(1,2,6)$. The patients without associated malignant carcinoma are younger with a lower level of GABABR-Abs, which is sometimes only presenting in the serum (6). Furthermore, non-paraneoplastic GABABRAbs encephalitis appear better respondence to the immunomodulatory treatment and consequently a better prognosis $(1,2,6)$. The paraneoplastic origin explained the poor outcomes among these patients. Tumor specialized treatment was an independent predictor of stabilization or improvement of the disease (OR 4.56; 95\% CI: 1.62-12.86), even the use of immunosuppressive (7). In the patient we studied, after laparoscopic radical nephrectomy plus immunotherapy, the re-examination of serum titer on GABAB receptor antibody significantly decreased. Furthermore, the neurological symptoms did not relapsed or worsen.

Evaluation of patients with suspected paraneoplastic neurological disorders is grounded on the knowledge of paraneoplastic syndromes, diagnosis of related tumors, and check antibodies of paraneoplastic. Paraneoplastic neurologic syndromes may have effect on either the peripheral or central nervous system, or both. Thus, the signs and symptoms of paraneoplastic syndromes are varying. Patients often exhibit diverse neurological manifestations, such as memory changes, seizures, movement disorders, like chorea, ataxia and dystonia, or even incidental coma. In numerous PNSs of central nervous system, the blood brain barrier remains intact, so the contrast enhancements of the affected regions rarely appeared. Central nervous system imaging manifestations may be invisible, and CSF analysis show a mild inflammation with high probability. Hence, diagnosis is highly dependent on clinical suspicion. Upon PNS is suspected, more systematic examinations for malignant carcinoma should be accomplished including a computed tomography scan of the abdomen, chest and pelvis. Nevertheless, if these preliminary reviews are negative, the whole-body PET imaging may be useful for locating the primary tumor. A unilateral or bilateral temporal lobe epileptic discharges may show through the EEG examination in patients with suspected PLE, revealing background activities, periodic lateralized epileptiform discharges, and even nonconvulsive status epilepticus (8). Paraneoplastic antibody testing plays a crucial part in the diagnosis of PNS and suggests the potential site of a latent cancer. Approximately 60 percent of patients with PNS of the NBS presented with the paraneoplastic antibodies positive (9). In up to $35 \%$ of cases, the GABAb-associated encephalitis can be paraneoplastic with SCLC most commonly (2). Our case presents a novelty in that anti-GABAb receptor encephalitis may be relevant to clear cell renal cell carcinoma.

Although there is an absence of proof-based recommendation in the matter of the diagnosis and treatment of patients with paraneoplastic syndromes related with cancer, immunotherapy is the most effective treatment 
and oncological treatment should be carried out as soon as possible. Our case indicates the combination of radical nephrectomy and immunotherapy improved neurological symptoms. Our patient demonstrated a longer survival time (more than 1.5 year) than the median survival (3 months) of patients with anti-GABAb receptor encephalitis associated with small cell lung cancer reported (2). The outcome of paraneoplastic encephalitis is related to the prognosis of the primary tumor. Prompt oncological treatment and immunotherapy can improve patients' prognosis.

\section{Acknowledgments}

We thanked Xin Jiang (native speaker (Canada)) for the proofreading of the paper.

Funding: None.

\section{Footnote}

Reporting Checklist: The authors have completed the CARE reporting checklist. Available at http://dx.doi.org/10.21037/ tcr-20-2769

Conflicts of Interest: All authors have completed the ICMJE uniform disclosure form (available at http://dx.doi. org/10.21037/tcr-20-2769). The authors have no conflicts of interest to declare.

Ethical Statement: The authors are accountable for all aspects of the work in ensuring that questions related to the accuracy or integrity of any part of the work are appropriately investigated and resolved. All procedures performed in studies involving human participants were in accordance with the ethical standards of the institutional and/or national research committee(s) and with the Helsinki Declaration (as revised in 2013). Written informed consent was obtained from the patient.

Open Access Statement: This is an Open Access article distributed in accordance with the Creative Commons
Attribution-NonCommercial-NoDerivs 4.0 International License (CC BY-NC-ND 4.0), which permits the noncommercial replication and distribution of the article with the strict proviso that no changes or edits are made and the original work is properly cited (including links to both the formal publication through the relevant DOI and the license). See: https://creativecommons.org/licenses/by-nc-nd/4.0/.

\section{References}

1. Lancaster E, Lai M, Peng X, et al. Antibodies to the GABA(B) receptor in limbic encephalitis with seizures: case series and characterisation of the antigen. Lancet Neurol 2010;9:67-76.

2. Höftberger R, Titulaer MJ, Sabater L, et al. Encephalitis and GABAB receptor antibodies: novel findings in a new case series of 20 patients. Neurology 2013;81:1500-6.

3. Gultekin SH, Rosenfeld MR, Voltz R, et al. Paraneoplastic limbic encephalitis: neurological symptoms, immunological findings and tumour association in 50 patients. Brain 2000;123:1481-94.

4. Benarroch EE. GABAB receptors: structure, functions, and clinical implications. Neurology 2012;78:578-84.

5. Lee $\mathrm{D}, \mathrm{Ha} M$, Hong $\mathrm{CM}$, et al. GABRQ expression is a potential prognostic marker for patients with clear cell renal cell carcinoma. Oncol Lett 2019;18:5731-8.

6. Dogan Onugoren M, Deuretzbacher D, Haensch CA, et al. Limbic encephalitis due to GABAB and AMPA receptor antibodies: a case series. J Neurol Neurosurg Psychiatry 2015;86:965-72.

7. Graus F, Keime-Guibert F, Reñe R, et al. Anti-Huassociated paraneoplastic encephalomyelitis: analysis of 200 patients. Brain 2001;124:1138-48.

8. van Sonderen A, Thijs RD, Coenders EC, et al. Anti-LGI1 encephalitis: Clinical syndrome and long-term follow-up. Neurology 2016;87:1449-56.

9. Giometto B, Grisold W, Vitaliani R, et al. Paraneoplastic neurologic syndrome in the PNS Euronetwork database: a European study from 20 centers. Arch Neurol 2010;67:330-5.
Cite this article as: Wang $\mathrm{H}$, Zhang W, Zhang X, Xu T, Xu K. Anti-GABAB receptor antibodies with autoimmune encephalitis in clear cell renal cell carcinoma: a case report. Transl Cancer Res 2021;10(2):1131-1134. doi: 10.21037/tcr-20-2769 